\title{
Corona: System Implications of Emerging Nanophotonic Technology
}

\author{
Dana Vantrease ${ }^{\dagger}$, Robert Schreiber ${ }^{\ddagger}$, Matteo Monchiero ${ }^{\ddagger}$, Moray McLaren ${ }^{\ddagger}$, Norman P. Jouppi ${ }^{\ddagger}$, \\ Marco Fiorentino $^{\ddagger}$, Al Davis ${ }^{\S}$, Nathan Binkert ${ }^{\ddagger}$, Raymond G. Beausoleil ${ }^{\ddagger}$, Jung Ho Ahn ${ }^{\ddagger}$ \\ ${ }^{\dagger}$ University of Wisconsin - Madison, ${ }^{\ddagger}$ Hewlett-Packard Laboratories, ${ }^{\S}$ University of Utah
}

\begin{abstract}
We expect that many-core microprocessors will push performance per chip from the 10 gigaflop to the 10 teraflop range in the coming decade. To support this increased performance, memory and inter-core bandwidths will also have to scale by orders of magnitude. Pin limitations, the energy cost of electrical signaling, and the non-scalability of chip-length global wires are significant bandwidth impediments. Recent developments in silicon nanophotonic technology have the potential to meet these off- and on-stack bandwidth requirements at acceptable power levels.

Corona is a 3D many-core architecture that uses nanophotonic communication for both inter-core communication and off-stack communication to memory or I/O devices. Its peak floating-point performance is 10 teraflops. Dense wavelength division multiplexed optically connected memory modules provide 10 terabyte per second memory bandwidth. A photonic crossbar fully interconnects its 256 low-power multithreaded cores at 20 terabyte per second bandwidth. We have simulated a 1024 thread Corona system running synthetic benchmarks and scaled versions of the SPLASH-2 benchmark suite. We believe that in comparison with an electrically-connected many-core alternative that uses the same on-stack interconnect power, Corona can provide 2 to 6 times more performance on many memoryintensive workloads, while simultaneously reducing power.
\end{abstract}

\section{Introduction}

Multi- and many-core architectures have arrived, and core counts are expected to double every 18 months [3]. As core count grows into the hundreds, the main memory bandwidth required to support concurrent computation on all cores will increase by orders of magnitude. Unfortunately, the ITRS roadmap [27] only predicts a small increase in pin count $(<2 x)$ over the next decade, and pin data rates are increasing slowly. This creates a significant bandwidth bottleneck. Similarly, the inability of on-chip networks to connect cores to memory or other cores at the required memory bandwidth poses a serious problem. Evidence suggests that many-core systems using electrical interconnects may not be able to meet these high bandwidth demands while maintaining acceptable performance, power, and area [19].

Nanophotonics offers an opportunity to reduce the power and area of off- and on-stack interconnects while meeting future system bandwidth demands. Optics is ideal for global communication because the energy cost is incurred only at the endpoints and is largely independent of length. Dense wavelength division multiplexing (DWDM) enables multiple single-wavelength communication channels to share a waveguide, providing a significant increase in bandwidth density. Recent nanophotonic developments demonstrate that waveguides and modulation/demodulation circuit dimensions are approaching electrical buffer and wire circuit dimensions [20].

Several benefits accrue when nanophotonics is coupled to emerging 3D packaging [1]. The 3D approach allows multiple die, each fabricated using a process well-suited to it, to be stacked and to communicate with through silicon vias (TSVs). Optics, logic, DRAM, non-volatile memory (e.g. FLASH), and analog circuitry may all occupy separate die and co-exist in the same 3D package. Utilizing the third dimension eases layout and helps decrease worst case wire lengths.

Corona is a nanophotonically connected 3D many-core NUMA system that meets the future bandwidth demands of data-intensive applications at acceptable power levels. Corona is targeted for a $16 \mathrm{~nm}$ process in 2017. Corona comprises 256 general purpose cores, organized in 64 fourcore clusters, and is interconnected by an all-optical, highbandwidth DWDM crossbar. The crossbar enables a cache coherent design with near uniform on-stack and memory communication latencies. Photonic connections to off-stack memory enables unprecedented bandwidth to large amounts of memory with only modest power requirements.

This paper presents key aspects of nanophotonic technology, and considers the implications for many-core processors. It describes the Corona architecture, and presents a performance comparison to a comparable, all-electrical many-core alternative. The contribution of this work is to show that nanophotonics is compatible with future CMOS technology, is capable of dramatically better communica- 
tion performance per unit area and energy, and can significantly improve the performance and utility of future manycore architectures.

\section{Photonic Technology}

Advances in silicon nanophotonics have made complete photonic on-stack communication networks a serious alternative to electrical networks. Photonic interconnects are interesting because they can be much more energy efficient than their electrical counterparts, especially at high speeds and long distances. In addition, the ability of optical fibers and waveguides to carry many information channels simultaneously increases interconnect bandwidth density significantly and eliminates the need for a large number of wires in order to achieve adequate bandwidth. Photonics dominates long-haul interconnects and is increasingly ubiquitous in metropolitan, storage, and local area networks. Photonic interconnects are becoming standard in data centers, and chip-to-chip optical links have been demonstrated [26]. This trend will naturally bring photonic interconnects into the chip stack, particularly since limited pin density and the power dissipation of global wires places significant constraints on performance and power.

A complete nanophotonic network requires waveguides to carry signals, light sources that provide the optical carrier, modulators that encode the data onto the carrier, photodiodes to detect the data, and injection switches that route signals through the network. It is imperative that the optical components be built in a single CMOS-compatible process to reduce the cost of introducing this new technology. Waveguides confine and guide light and need two optical materials: a high refraction index material to form the core of the waveguide and a low index material that forms the cladding. We use crystalline silicon (index $\approx 3.5$ ) and silicon oxide (index $\approx 1.45$ ). Both are commonly used in CMOS processes. A silicon oxide waveguide has typical cross-sectional dimensions of $\approx 500 \mathrm{~nm}$ with a wall thickness of least $1 \mu \mathrm{m}$. These waveguides have been shown to be able to carry light with losses on the order of $2-3 \mathrm{~dB} / \mathrm{cm}$ and can be curved with bend radii on the order of $10 \mu \mathrm{m}$.

In order to communicate rather than simply illuminate, we must introduce a second material to absorb light and convert the light into an electric signal. Germanium is a natural choice: it is already being used in CMOS processes and has a significant photo-absorption between 1.1 and $1.5 \mu \mathrm{m}$. While it is possible to induce strains in the crystalline structure of $\mathrm{Ge}$ to extend its absorption window into the 1.55 $\mu \mathrm{m}$ window commonly used for long distance fiber communication, it is easier to rely on unstrained Ge and use light around $1.3 \mu \mathrm{m}$. This wavelength is still compatible with fiber transmission, which is an important characteristic for off-stack networks that will need to use some kind of waveguide or optical fiber to connect different optically enabled stacks.

The third element is a light source, and a laser is the obvious choice. A laser's narrow linewidth allows one to pack many communication channels in a single waveguide, thus increasing bandwidth. There are two possible ways to encode data in laser light. The first method uses direct modulation of the laser, where the laser is switched on and off to represent digital $1 \mathrm{~s}$ and $0 \mathrm{~s}$. The second method uses a continuous-wave $(\mathrm{CW})$ laser and a separate modulator to achieve the required modulation. To achieve the high modulation speeds that would make on-stack interconnects practical (typically $10 \mathrm{~Gb} / \mathrm{s}$ data rates) one would need to use vertical cavity semiconductor lasers (VCSELs) for direct modulation. Since VCSELs are built using III-V compound semiconductors, they cannot be easily integrated in a CMOS-compatible process. On-stack mode-locked lasers are an interesting separate modulation alternative. A modelocked laser generates a comb of phase-coherent wavelengths at equally spaced wavelengths. On-stack modelocked lasers have been recently demonstrated [16]. It is expected that one such laser could provide 64 wavelengths for a DWDM network.

For separate modulation, external modulators are required. In a DWDM network it is preferable to have wavelength-selective modulators that can modulate a single wavelength in a multi-wavelength channel. This simplifies the topology of the network and increases its flexibility. Wavelength-selective silicon modulators with modulation rates in excess of $10 \mathrm{~Gb} / \mathrm{s}$ have recently been demonstrated [35]. These modulators are based on ring resonators built using an SOI waveguide in a ring with diameter of 3$5 \mu \mathrm{m}$ (Figure 1(a)). Depending on their construction, they may serve to modulate, inject, or detect the light.

To modulate light, a ring is placed next to a waveguide. A fraction of the light will be coupled and circulate inside the ring. For some wavelengths, the length of the ring circumference will be equal to an integer number of wavelengths. In this resonance condition, the light in the ring will be enhanced by interference while the light transmitted by the waveguide will be suppressed (Figure 1(b)). In a properly coupled ring, the waveguide's light will be suppressed and the rings' light will be eventually lost due to the bend in the ring and scattering from imperfections. The wavelength depends primarily on the index of refraction of the materials that constitute the ring. We can further fine-tune the wavelength (or index of refraction) by injecting charge into the ring or changing the temperature of the ring. This brings the ring in and out of resonance. The first method is commonly used for fast modulation while the second can be used for slow tuning.

The same ring structure can be used to inject a single wavelength from one waveguide to another. If the ring is 
a)

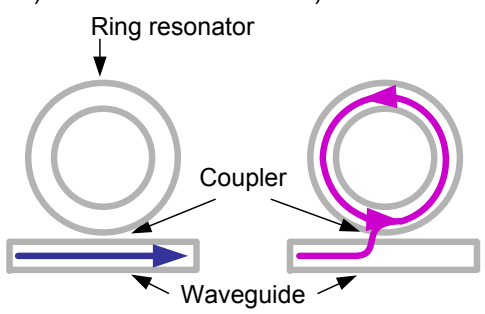

c)

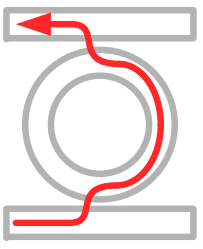

d)

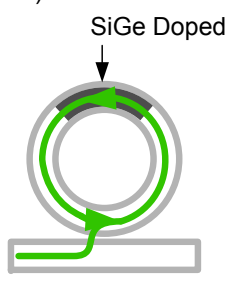

e)

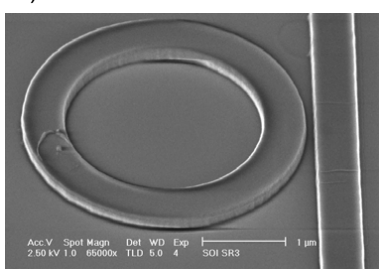

Figure 1: Schematic Top View of Nanophotonic Building Blocks. (a) Off-resonance Modulator. The ring resonator is coupled to a waveguide through evanescent coupling. Off-resonance wavelengths are transmitted through. (b) On-resonance Modulator. A resonant wavelength is coupled in the ring and eventually gets attenuated by losses. A negligible amount of light is transmitted through due to destructive interference. By switching between the on- and off-resonance state one can achieve modulation (or diversion) of a continuous-wave laser. (c) Injector. A resonant wavelength in the input (lower) waveguide is coupled into the ring and out through the output (top) waveguide. (d) Detector. A resonant wavelength is coupled in the ring and absorbed by a SiGe detector coupled to the ring. (e) SEM image of a $3 \mu \mathrm{m}$ diameter resonator ring. Image courtesy of Qianfan Xu, HP Labs Palo Alto.

placed between two waveguides and the coupling between the ring and the waveguides is properly chosen, the wavelength resonating within the ring will be transferred from one waveguide to the other. By bringing the ring in and out of resonance, a frequency-selective switch injector (Figure $1(\mathrm{c}))$ can be realized.

A ring resonator can also be used as a wavelengthselective detector (Figure 1(d)). If germanium is included in the resonator, the resonant wavelength will be absorbed by the germanium and it will generate a photocurrent while non-resonant wavelengths will be transmitted. An advantage of this scheme is that because the resonant wavelength will circulate many times in the ring, only very small absorption rates (less than $1 \%$ per pass) will be needed and therefore a small detector will be sufficient. This brings the capacitance of the detector down to $\approx 1 \mathrm{fF}$ and removes the need for power-hungry trans-impedance amplifiers.

A final component that is not necessary for photonic networks but that we find useful is a broadband splitter. A broadband splitter distributes power and data by splitting the signal between two waveguides. It diverts a fixed fraction of optical power from all wavelengths of one waveguide and injects them onto another waveguide. Other than a drop in strength, the unsplit portion of the signal is unaffected by the splitter.

While most of the individual components of a DWDM on-stack network have been demonstrated [16, 35], a number of important problems remain. Foremost among these is the necessity to integrate a large number of devices in a single chip. It will be necessary to analyze and correct for the inevitable fabrication variations to minimize device failures and maximize yield. A large effort will also be needed to design the analog electronics that drive and control the optical devices in a power-efficient way. While significant research is still necessary, we believe that DWDM photonic networks offer a credible answer to the challenges posed by the increasing bandwidth needs of many-core architectures.

\section{The Corona Architecture}

Corona is a tightly coupled, highly parallel NUMA system. As NUMA systems and applications scale, it becomes more difficult for the programmer, compiler, and runtime system to manage the placement and migration of programs and data. We try to lessen the burden with homogeneous cores and caches, a crossbar interconnect that has nearuniform latency, a fair interconnect arbitration protocol, and high (one byte per flop) bandwidth between cores and from caches to memory.

The architecture is made up of 256 multithreaded inorder cores and is capable of supporting up to 1024 threads simultaneously, providing up to 10 teraflops of computation, up to 20 terabytes per second (TB/s) of on-stack bandwidth, and up to $10 \mathrm{~TB} / \mathrm{s}$ of off-stack memory bandwidth.

Figure 2 gives a conceptual view of the system while Figure 3 provides a sample layout of the system including the waveguides that comprise the optical interconnect (Section 3.2), the optical connection to memory (Section 3.3), and other optical components.

\subsection{Cluster Architecture}

Each core has a private L1 instruction and data cache, and all four cores share a unified L2 cache. A hub routes message traffic between the L2, directory, memory controller, network interface, optical bus, and optical crossbar. Figure 2(b) shows the cluster configuration, while the upper left hand insert in Figure 3 shows its approximate floorplan.

Because Corona is an architecture targeting future high throughput systems, our exploration and evaluation of the 


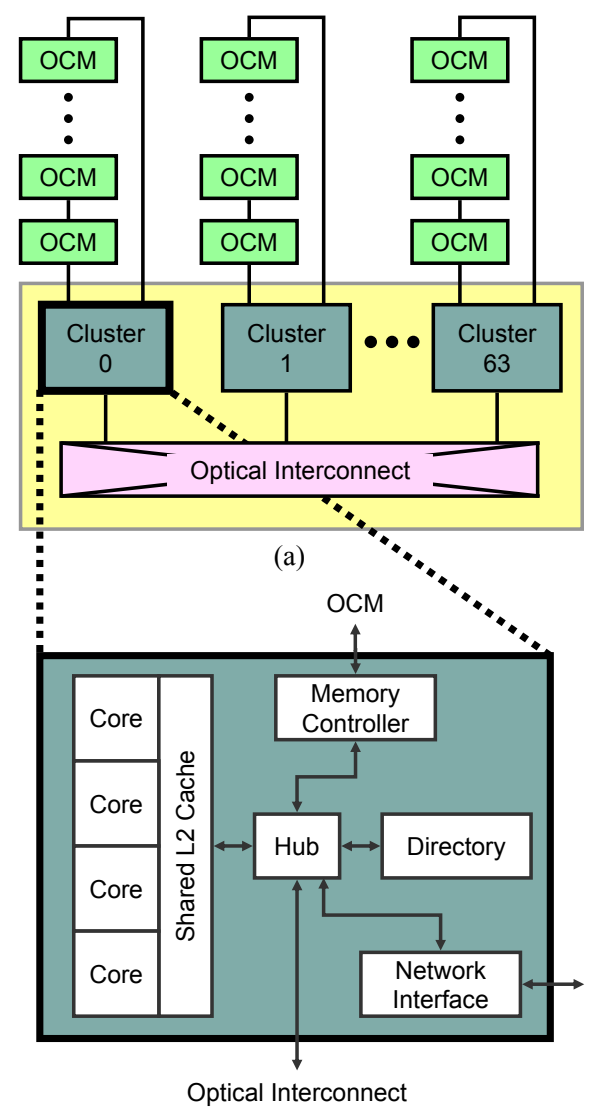

(b)

Figure 2: Architecture Overview

architecture is not targeted at optimal configuration of the clusters' subcomponents (such as their branch prediction schemes, number of execution units, cache sizes, and cache policies). Rather, the clusters' design parameters (Table 1) represent reasonably modest choices for a highperformance system targeted at a $16 \mathrm{~nm}$ process in 2017.

\subsubsection{Cores}

The core choice is primarily motivated by power; with hundreds of cores, each one will need to be extremely energy efficient. We use dual-issue, in-order, four-way multithreaded cores.

Power analysis has been based on the Penryn [13] (desktop and laptop market segments) and the Silverthorne [12] (low-power embedded segment) cores. Penryn is a singlethreaded out-of-order core supporting 128-bit SSE4 instructions. Power per core has been conservatively reduced by $5 x$ (compared to the $6 x$ predictions in [3]) and then increased by $20 \%$ to account for differences in the quadthreaded Corona. Silverthorne is a dual-threaded in-order 64-bit design where power and area have been increased to account for the Corona architectural parameters. Direc-

\begin{tabular}{l|r} 
Resource & Value \\
\hline Number of clusters & 64 \\
Per-Cluster: & \\
L2 cache size/assoc & 4 MB/16-way \\
L2 cache line size & 64 B \\
L2 coherence & MOESI \\
Memory controllers & 1 \\
Cores & 4 \\
Per-Core: & \\
L1 ICache size/assoc & $16 \mathrm{~KB} / 4$-way \\
L1 DCache size/assoc & $32 \mathrm{~KB} / 4$-way \\
L1 I \& D cache line size & 64 B \\
Frequency & 5 GHz \\
Threads & 4 \\
Issue policy & In-order \\
Issue width & 2 \\
64 b floating point SIMD width & 4 \\
Fused floating point operations & Multiply-Add
\end{tabular}

Table 1: Resource Configuration

tory and L2 cache power has been calculated using CACTI 5 [30]. Hub and memory controller power estimates are based on synthesized $65 \mathrm{~nm}$ designs and Synopsis Nanosim power values scaled to $16 \mathrm{~nm}$. Total processor, cache, memory controller and hub power for the Corona design is expected to be between 82 watts (Silverthorne based) and 155 watts (Penryn based).

Area estimates are based on pessimistically scaled Penryn and Silverthorne designs. We estimate that an in-order Penryn core will have one-third the area of the existing outof-order Penryn. This estimate is consistent with the current core-size differences in $45 \mathrm{~nm}$ for the out-of-order Penryn and the in-order Silverthorne, and is more conservative than the $5 x$ area reduction reported by Asanovic et al. [3]. We then assume a multithreading area overhead of $10 \%$ as reported in Chaudry et al. [7]. Total die area for the processor and L1 die is estimated to be between $423 \mathrm{~mm}^{2}$ (Penryn based) and $491 \mathrm{~mm}^{2}$ (Silverthorne based). The discrepancy between these estimates is likely affected by the 6transistor Penryn L1 cache cell design vs. the 8-transistor Silverthorne L1 cache cell.

\subsubsection{On-Stack Memory}

Corona employs a MOESI directory protocol. The protocol is backed by a single broadcast bus (see Section 3.2.2), which is used to quickly invalidate a large pool of sharers with a single message. The coherence scheme was included for purposes of die size and power estimation, but has not yet been modeled in the system simulation. Nonetheless, we believe that our performance simulations provide a sensible first-order indication of Corona's potential.

The Corona architecture has one memory controller per cluster. Associating the memory controller with the cluster 


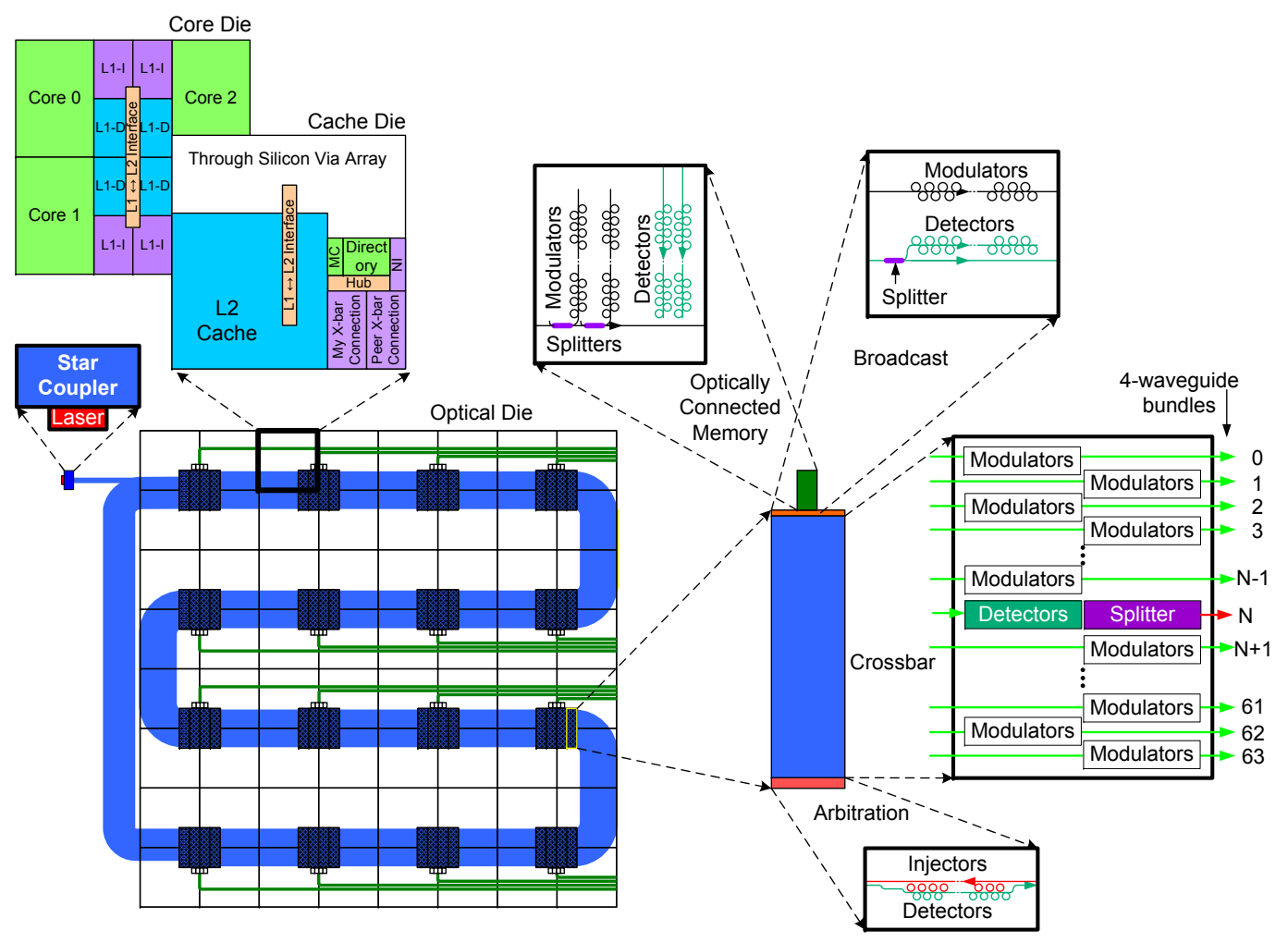

Figure 3: Layout with Serpentine Crossbar and Resonator Ring Detail

ensures that the memory bandwidth grows linearly with increased core count, and it provides local memory accessible with low latency. Photonics connects the memory controller to off-stack memory as detailed in Section 3.3.

Network interfaces, similar to the interface to off-stack main memory, provide inter-stack communication for larger systems using DWDM interconnects.

\subsection{On-Stack Photonic Interconnect}

Corona's 64 clusters communicate through an optical crossbar and occasionally an optical broadcast ring. Both are managed using optical tokens. Several messages of different sizes may simultaneously share any communication channel, allowing for high utilization.

Table 2 summarizes the interconnects' optical component requirements (power waveguides and I/O components are omitted). Based on existing designs, we estimate that the photonic interconnect power (including the power dissipated in the analog circuit layer and the laser power in the photonic die) to be $39 \mathrm{~W}$.

\subsubsection{Optical Crossbar}

Each cluster has a designated channel that address, data, and coherence messages share. Any cluster may write to a given channel, but only a single fixed cluster may read from

\begin{tabular}{l|r|r} 
Photonic Subsystem & Waveguides & Ring Resonators \\
\hline Memory & 128 & $16 \mathrm{~K}$ \\
Crossbar & 256 & $1024 \mathrm{~K}$ \\
Broadcast & 1 & $8 \mathrm{~K}$ \\
Arbitration & 2 & $8 \mathrm{~K}$ \\
Clock & 1 & 64 \\
\hline Total & 388 & $\approx 1056 \mathrm{~K}$
\end{tabular}

Table 2: Optical Resource Inventory

the channel. A fully-connected $64 \times 64$ crossbar can be realized by replicating this many-writer single-reader channel 64 times, adjusting the assigned "reader" cluster with each replication.

The channels are each 256 wavelengths, or 4 bundled waveguides, wide. When laid out, the waveguide bundle forms a broken ring that originates at the destination cluster (the channel's home cluster), is routed past every other cluster, and eventually terminates back at its origin. Light is sourced at a channel's home by a splitter that provides all wavelengths of light from a power waveguide. Communication is unidirectional, in cyclically increasing order of cluster number.

A cluster sends to another cluster by modulating the light on the destination cluster's channel. Figure 4 illustrates the 

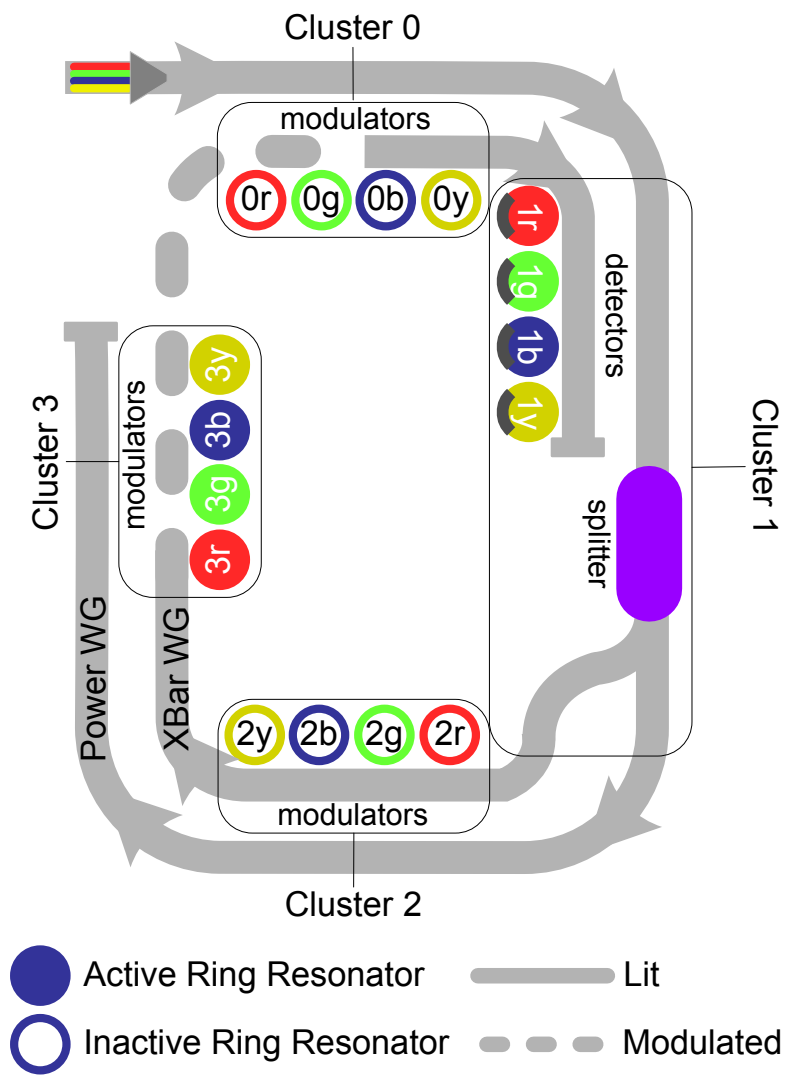

Figure 4: A Four Wavelength Data Channel Example. The home cluster (cluster 1) sources all wavelengths of light $(\mathrm{r}, \mathrm{g}, \mathrm{b}, \mathrm{y})$. The light travels clockwise around the crossbar waveguides. It passes untouched by cluster 2's inactive (offresonance) modulators. As it passes by cluster 3's active modulators, all wavelengths are modulated to encode data. Eventually, cluster 1's detectors sense the modulation, at which point the waveguide terminate.

conceptual operation of a four-wavelength channel. Modulation occurs on both clock edges, so that each of the wavelengths signals at $10 \mathrm{~Gb} / \mathrm{s}$, yielding a per-cluster bandwidth of 2.56 terabits per second $(\mathrm{Tb} / \mathrm{s})$ and a total crossbar bandwidth of $20 \mathrm{~TB} / \mathrm{s}$.

A wide phit with low modulation time keeps latency to a minimum, which is critical to ensuring the in-order cores minimize stall time. A 64-byte cache line can be sent (256 bits in parallel twice per clock) in one $5 \mathrm{GHz}$ clock. The propagation time is at most 8 clocks and is determined by a combination of the source's distance from the destination and the speed of light in a silicon waveguide (approximately $2 \mathrm{~cm}$ per clock). Because messages, such as cache lines, are localized to a small portion of the bundle's length, a bundle may have multiple back-to-back messages in transit simultaneously.

Corona uses optical global distribution of the clock in or- der to avoid the need for signal retiming at the destination. A clock distribution waveguide parallels the data waveguide, with the clock signal traveling clockwise with the data signals. This means that each cluster is offset from the previous cluster by approximately $1 / 8$ th of a clock cycle. A cluster's electrical clock is phase locked to the arriving optical clock. Thus, input and output data are in phase with the local clock; this avoids costly retiming except when the serpentine wraps around.

\subsubsection{Optical Broadcast Bus}

In the MOESI coherency protocol, when a shared block is invalidated, an invalidate message must be multicast to all sharers. For unicast interconnects, such as Corona's crossbar, the multicast is translated into several unicast messages. These unicast messages cause network congestion and may harm performance [14].

We avoid redundant unicast invalidates by augmenting our system with a broadcast bus. The broadcast bus is a single waveguide that passes by each cluster twice in a coiled, or spiral-like, fashion. The upper right hand corner of Figure 3 gives a cluster-centric view of the bus' details. The light is sourced from one endpoint of the coil. On the light's first pass around the coil, clusters modulate the light to encode invalidate messages. On the light's second pass, the invalidate messages become active, that is, clusters may read the messages and snoop their caches. To do this, each cluster has a splitter that transfers a fraction of the light from the waveguide to a short dead-end waveguide that is populated with detectors.

In addition to broadcasting invalidates, the bus' functionality could be generalized for other broadcast applications, such as bandwidth adaptive snooping [22] and barrier notification.

\subsubsection{Arbitration}

The crossbar and broadcast bus both require a conflict resolution scheme to prevent two or more sources from concurrently sending to the same destination. Our solution is a distributed, all optical, token-based arbitration scheme that fairly allocates the available interconnect channels to clusters. Token ring arbitration is naturally distributed and has been used in token ring LAN systems [2]. Its asynchronous acquire-and-release nature tolerates variability in request arrival time, message modulation time, and message propagation time. Figure 5 demonstrates a photonic version of this approach.

In our implementation, a token conveys the right to send data on a given channel. The one-bit token is represented by the presence of a short signal in a specific wavelength on an arbitration waveguide. For the broadcast bus, we use one wavelength. For the crossbar, we use 64 wavelengths, in one to one correspondence with the 64 crossbar channels. 

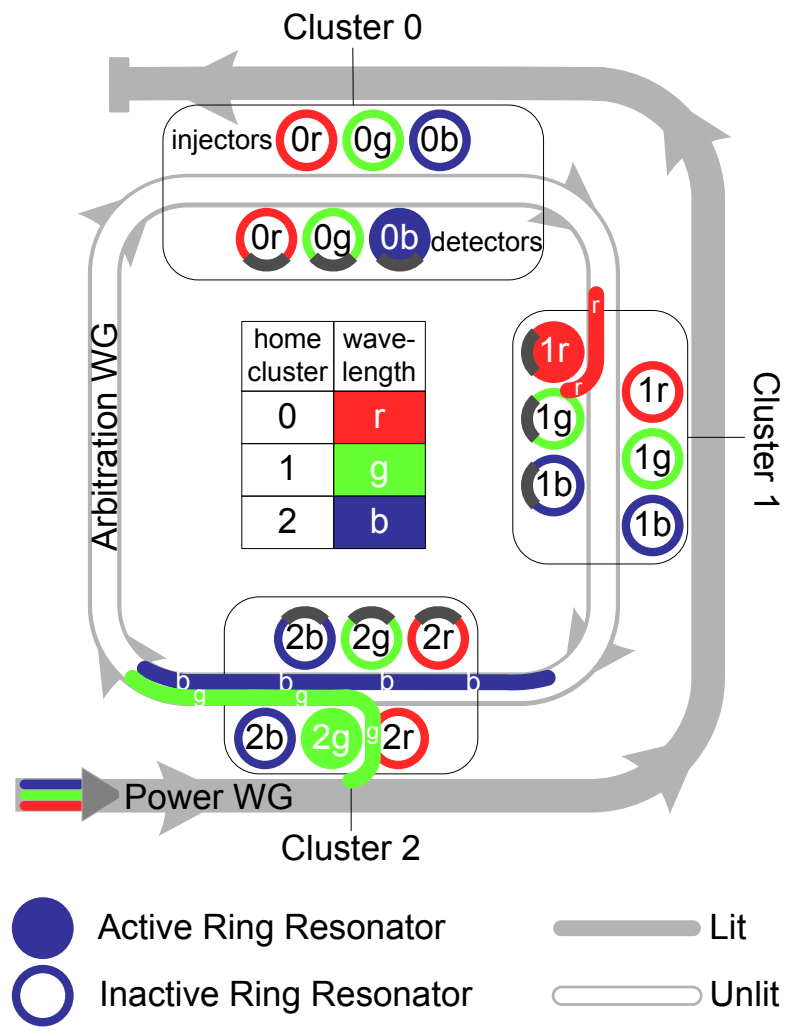

Figure 5: Optical Token Arbitration Example. 3 wavelengths are used to arbitrate for 3 channels. The channel-totoken (home cluster-to-wavelength) mapping is shown in the embedded table. In this depiction, all tokens are in transit (i.e. all channels are free). Cluster 0 is requesting cluster 2 (blue), will soon divert blue, and will then begin transmitting on cluster 2's channel. Cluster 1 is requesting cluster 0 (red), has nearly completed diverting red, and will soon begin transmitting on cluster 0's channel. Cluster 2 is re-injecting green (cluster 1) because it has just finished transmitting on cluster 1's channel. (Note: Detectors are positioned to prevent a cluster from reacquiring a self-injected token until it has completed one revolution around the ring.)

When a cluster finishes sending a message on a channel, it releases the channel by activating its injector and reintroducing the token onto the arbitration waveguide. The token travels in parallel with the tail of the most recently transmitted message. Each cluster is equipped with an array of fixed-wavelength detectors that are capable of diverting (obtaining) any token. If a token is diverted, the light is completely removed from the arbitration waveguide to provide an exclusive grant for the corresponding channel. Each cluster will absorb and regenerate its channel token to ensure that it remains optically sound even after many trips around the ring without any "takers."

This scheme fairly allocates the channels in a roundrobin order. When many clusters want the same channel and contention is high, token transfer time is low and channel utilization is high. However when contention is low, a cluster may wait as long as 8 processor clock cycles for an uncontested token.

\subsection{Optically Connected Memory}

One design goal is to scale main memory bandwidth to match the growth in computational power. Maintaining this balance ensures that the performance of the system is not overly dependent on the cache utilization of the application. Our target external memory bandwidth for a 10 teraflop processor is $10 \mathrm{~TB} / \mathrm{s}$. Using an electrical interconnect to achieve this performance would require excessive power; over $160 \mathrm{~W}$ assuming $2 \mathrm{~mW} / \mathrm{Gb} / \mathrm{s}$ [25] interconnect power. Instead, we use a nanophotonic interconnect that has high bandwidth and low power. The same channel separations and data rates that are used on the internal interconnect network can also be used for external fiber connections. We estimate the interconnect power to be $0.078 \mathrm{~mW} / \mathrm{Gb} / \mathrm{s}$, which equates to a total memory system power of approximately $6.4 \mathrm{~W}$.

Each of the 64 memory controllers connects to its external memory by a pair of single-waveguide, 64-wavelength DWDM links. The optical network is modulated on both edges of the clock. Hence each memory controller provides $160 \mathrm{~GB} / \mathrm{s}$ of off-stack memory bandwidth, and all memory controllers together provide $10 \mathrm{~TB} / \mathrm{s}$.

This allows all communication to be scheduled by the memory controller with no arbitration. Each external optical communication link consists of a pair of fibers providing half duplex communication between the CPU and a string of optically connected memory (OCM) modules. The link is optically powered from the chip stack; after connecting to the OCMs, each outward fiber is looped back as a return fiber. Although the off-stack memory interconnect uses the same modulators and detectors as the on-stack interconnects, the communication protocols differ. Communication between processor and memory is master/slave, as opposed to peer-to-peer. To transmit, the memory controller modulates the light and the target module diverts a portion of the light to its detectors. To receive, the memory controller detects light that the transmitting OCM has modulated on the return fiber. Because the memory controller is the master, it can supply the necessary unmodulated power to the transmitting OCM.

Figure 6(a) shows the 3D stacked OCM module, built from custom DRAM die and an optical die. The DRAM die is organized so that an entire cache line is read or written from a single mat. 3D stacking is used to minimize the delay and power in the interconnect between the optical fiber loop and the DRAM mats. The high-performance optical interconnect allows a single mat to quickly provide all the data for an entire cache line. In contrast, current electri- 


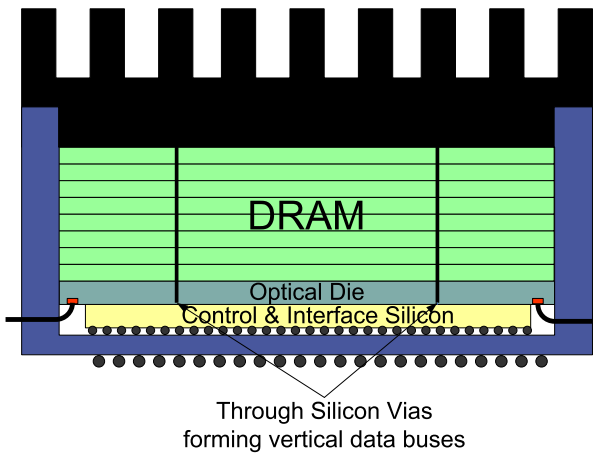

(a)

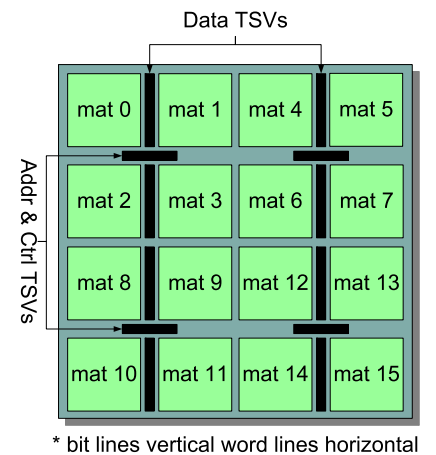

(b)

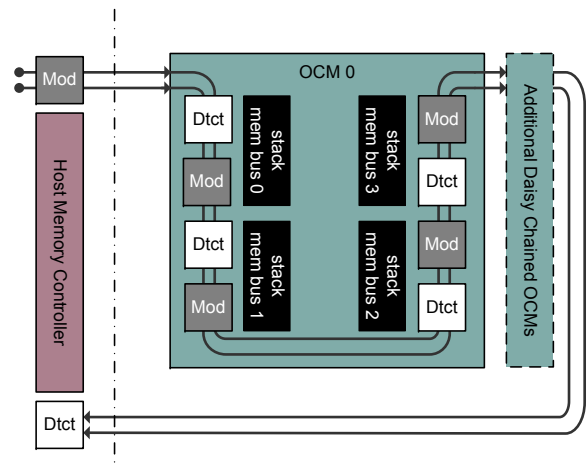

(c)

Figure 6: Schematic View of Optically Connected Memory. (a) 3D die stack. The stack has one optical die and multiple DRAM dies. (b) DRAM die floorplan. Each quadrant is independent, and could also be constructed from four independent die. (c) OCM expansion. The light travels from the processor, through one or more OCMs, finally looping back to the processor.

cal memory systems and DRAMs activate many banks on many die on a DIMM, reading out tens of thousands of bits into an open page. However, with highly interleaved memory systems and a thousand threads, the chances of the next access being to an open page are small. Corona's DRAM architecture avoids accessing an order of magnitude more bits than are needed for the cache line, and hence consumes less power in its memory system.

Corona supports memory expansion by adding additional OCMs to the fiber loop as shown in Figure 6(c). Expansion adds only modulators and detectors and not lasers, so the incremental communication power is small. As the light passes directly through the OCM without buffering or retiming, the incremental delay is also small, so that the memory access latency is similar across all modules. In contrast, a serial electrical scheme, such as FBDIMM, would typically require the data to be resampled and retransmitted at each module, increasing the communication power and access latency.

\subsection{Chip Stack}

Figure 7 illustrates the Corona 3D die stack. Most of the signal activity, and therefore heat, are in the top die (adjacent to the heat sink) which contains the clustered cores and L1 caches. The processor die is face-to-face bonded with the L2 die, providing direct connection between each cluster and its L2 cache, hub, memory controller, and directory. The bottom die contains all of the optical structures (waveguides, ring resonators, detectors, etc.) and is face-to-face bonded with the analog electronics which contain detector circuits and control ring resonance and modulation.

All of the L2 die components are potential optical communication end points and connect to the analog die by signal through silicon vias (sTSVs). This strategy minimizes the layout impact since most die-to-die signals are carried in

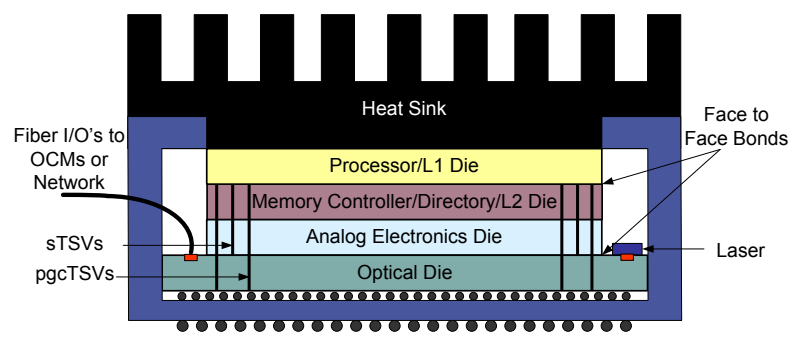

Figure 7: Schematic Side View of 3D Package

the face-to-face bonds. External power, ground, and clock vias (pgcTSVs) are the only TSVs that must go through three die to connect the package to the top two digital layers. The optical die is larger than the other die in order to expose a mezzanine to permit fiber attachments for $\mathrm{I} / \mathrm{O}$ and OCM channels and external lasers.

\section{Experimental Setup}

We subject our architecture to a combination of synthetic and realistic workloads that were selected with an eye to stressing the on-stack and memory interconnects. Synthetic workloads stress particular features and aspects of the interconnects. The SPLASH-2 benchmark suite [34] indicates their realistic performance. The SPLASH-2 applications are not modified in their essentials. We use larger datasets when possible to ensure that each core has a nontrivial workload. Because of a limitation in our simulator, we needed to replace implicit synchronization via semaphore variables with explicit synchronization constructs. In addition, we set the L2 cache size to $256 \mathrm{~KB}$ to better match our simulated benchmark size and duration when scaled to expected system workloads. A summary of the workload setup is described in Table 3. 


\begin{tabular}{l|l|r} 
Synthetic & & \# Network \\
Benchmark & Description & $1 \mathrm{M}$ \\
\hline Uniform & Uniform random & $1 \mathrm{M}$ \\
Hot Spot & All clusters to one cluster & $1 \mathrm{M}$ \\
Tornado & Cluster $(i, j)$ to cluster & \\
& $((i+\lfloor k / 2\rfloor-1) \% k$, & \\
& $(j+\lfloor k / 2\rfloor-1) \% k)$, & \\
Transpose & Cluster $k=$ network's radix & \\
&
\end{tabular}

\begin{tabular}{l|c|r} 
SPLASH-2 & Data Set & \# Network \\
Benchmark & Experimental (Default) & $7.2 \mathrm{M}$ \\
\hline Barnes & $64 \mathrm{~K}$ particles $(16 \mathrm{~K})$ & $0.6 \mathrm{M}$ \\
Cholesky & tk29.O (tk15.O) & $176 \mathrm{M}$ \\
FFT & $16 \mathrm{M}$ points $(64 \mathrm{~K})$ & $1.8 \mathrm{M}$ \\
FMM & $1 \mathrm{M}$ particles $(16 \mathrm{~K})$ & $34 \mathrm{M}$ \\
LU & $2048 \times 2048$ matrix $(512 \times 512)$ & $240 \mathrm{M}$ \\
Ocean & $2050 \times 2050$ grid $(258 \times 258)$ & $4.2 \mathrm{M}$ \\
Radiosity & roomlarge $($ room $)$ & $189 \mathrm{M}$ \\
Radix & $64 \mathrm{M}$ integers $(1 \mathrm{M})$ & $0.7 \mathrm{M}$ \\
Raytrace & balls4 $($ car $)$ & $3.6 \mathrm{M}$ \\
Volrend & head (head) & $3.2 \mathrm{M}$ \\
Water-Sp & $32 \mathrm{~K}$ molecules $(512)$ &
\end{tabular}

Table 3: Benchmarks and Configurations

The simulation infrastructure is split into two independent parts: a full system simulator for generating L2 miss memory traces and a network simulator for processing these traces. A modified version of the HP Labs' COTSon simulator [11] generates the traces. (COTSon is based on AMD's SimNow simulator infrastructure.) Each application is compiled with gcc 4.1, using -O3 optimization, and run as a single 1024-threaded instance. We are able to collect multithreaded traces by translating the operating system's threadlevel parallelism into hardware thread-level parallelism. In order to keep the trace files and network simulations manageable, the simulators do not tackle the intricacies of cache coherency between clusters.

The network simulator reads the traces and processes them in the network subsystem. The traces consist of L2 misses and synchronization events that are annotated with thread id and timing information. In the network simulator, L2 misses go through a request-response, on-stack interconnect transaction and an off-stack memory transaction. The simulator, which is based on the M5 framework [4], takes an trace-driven approach to processing memory requests. The MSHRs, hub, interconnect, arbitration, and memory are all modeled in detail with finite buffers, queues, and ports. This enforces bandwidth, latency, back pressure, and capacity limits throughout.

In the simulation, our chief goal is to understand the performance implications of the on-stack network and the off-

\begin{tabular}{l|c|c} 
Resource & OCM & ECM \\
\hline Memory controllers & 64 & 64 \\
External connectivity & 256 fibers & 1536 pins \\
Channel width & $128 \mathrm{~b}$ half duplex & $12 \mathrm{~b}$ full duplex \\
Channel data rate & $10 \mathrm{~Gb} / \mathrm{s}$ & $10 \mathrm{~Gb} / \mathrm{s}$ \\
Memory bandwidth & $10.24 \mathrm{~TB} / \mathrm{s}$ & $0.96 \mathrm{~TB} / \mathrm{s}$ \\
Memory latency & $20 \mathrm{~ns}$ & $20 \mathrm{~ns}$
\end{tabular}

Table 4: Optical vs Electrical Memory Interconnects

stack memory design. Our simulator has three network configuration options:

- XBar - An optical crossbar (as described in Section 3.2), with bisection bandwidth of $20.48 \mathrm{~TB} / \mathrm{s}$, and maximum signal propagation time of 8 clocks.

- HMesh - An electrical 2D mesh with bisection bandwidth $1.28 \mathrm{~TB} / \mathrm{s}$ and per hop signal latency (including forwarding and signal propagation time) of 5 clocks.

- LMesh - An electrical 2D mesh with bisection bandwidth $0.64 \mathrm{~TB} / \mathrm{s}$ and per hop signal latency (including forwarding and signal propagation time) of 5 clocks.

The two meshes employ dimension-order wormhole routing [9]. We estimated a worst-case power of $26 \mathrm{~W}$ for the optical crossbar. Since many components of the optical system power are fixed (e.g., laser, ring trimming, etc.), we conservatively assumed a continuous power of $26 \mathrm{~W}$ for the XBar. We assumed an electrical energy of $196 \mathrm{pJ}$ per transaction per hop, including router overhead. This aggressively assumes low swing busses and ignores all leakage power in the electrical meshes.

We also simulate two memory interconnects, the OCM interconnect (as described in Section 3.3) plus an electrical interconnect:

- OCM - Optically connected memory; off-stack memory bandwidth is $10.24 \mathrm{~TB} / \mathrm{s}$, memory latency is $20 \mathrm{~ns}$.

- ECM - Electrically connected memory; off-stack memory bandwidth is $0.96 \mathrm{~TB} / \mathrm{s}$, memory latency is $20 \mathrm{~ns}$.

The electrical memory interconnect is based on the ITRS roadmap, according to which it will be impossible to implement an ECM with performance equivalent to the proposed OCM. Table 4 contrasts the memory interconnects.

We simulate five combinations: XBar/OCM (i.e. Corona), HMesh/OCM, LMesh/OCM, HMesh/ECM, and LMesh/ECM. These choices highlight, for each benchmark, the performance gain, if any, due to faster memory and due to faster interconnect. We ran each simulation for a predetermined number of network requests (L2 misses). These miss counts are shown in Table 3. 


\section{Performance Evaluation}

For the five system configurations, Figure 8 shows performance relative to the realistic, electrically connected LMesh/ECM system.

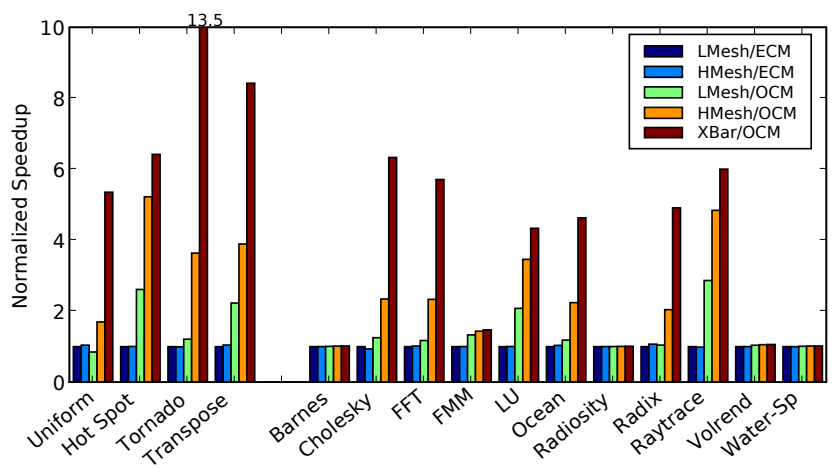

Figure 8: Normalized Speedup

We can form a few hypotheses based on the synthetic benchmarks. Evidently, with low memory bandwidth, the high-performance mesh adds little value. With fast OCM, there is very substantial performance gain over ECM systems, when using the fast mesh or the crossbar interconnect, but much less gain if the low performance mesh is used. Most of the performance gain made possible by OCM is realized only if the crossbar interconnect is used. In the exceptional case, Hot Spot, memory bandwidth remains the performance limiter (because all the memory traffic is channeled through a single cluster); hence there is less pressure on the interconnect. Overall, by moving to an OCM from an ECM in systems with an HMesh, we achieve a geometric mean speedup of 3.28. Adding the photonic crossbar can provide a further speedup of 2.36 on the synthetic benchmarks.

For the SPLASH-2 applications, we find that in four cases (Barnes, Radiosity, Volrend, and Water-Sp) the LMesh/ECM system is fully adequate. These applications perform well due to their low cache-miss rates and consequently low main memory bandwidth demands. FMM is quite similar to these. The remaining applications are memory bandwidth limited on ECM-based systems. For Cholesky, FFT, Ocean, and Radix, fast memory provides considerable benefits, which are realized only with the fast crossbar. LU and Raytrace are like Hot Spot: while OCM gives most of the significant speedup, some additional benefit derives from the use of the fast crossbar. We posit below a possible reason for the difference between Cholesky, FFT, Ocean, and Radix on the one hand, and LU and Raytrace on the other, when examining the bandwidth and latency data. These observations are generally consistent with the detailed memory traffic measurements reported by Woo et al. [34]. Overall, replacing an ECM with an OCM in a system using an HMesh can provide a geometric mean speedup of 1.80. Adding the photonic crossbar can provide a further speedup of 1.44 on the SPLASH-2 applications.

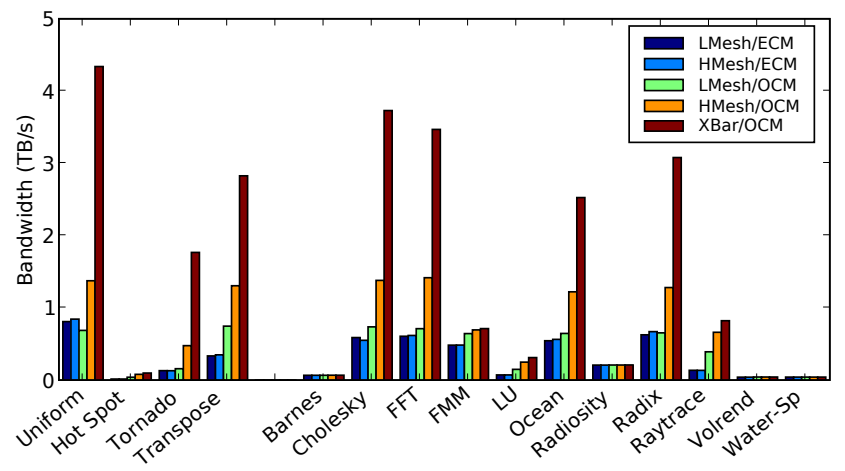

Figure 9: Achieved Bandwidth

Figure 9 shows the actual rate of of communication with main memory. The four low bandwidth applications that perform well on the LMesh/ECM configuration are those with bandwidth demands lower than that provided by ECM. FMM needs somewhat more memory bandwidth than ECM provides. Three of the synthetic tests and four of the applications have very high bandwidth and interconnect requirements, in the $2-5 \mathrm{~TB} / \mathrm{s}$ range; these benefit the most from the XBar/OCM configuration. LU and Raytrace do much better on OCM systems than ECM, but do not require much more bandwidth than ECM provides. They appear to benefit mainly from the improved latency offered by XBar/OCM. We believe that this is due to bursty memory traffic in these two applications. Analysis of the LU code shows that many threads attempt to access the same remotely stored matrix block at the same time, following a barrier. In a mesh, this oversubscribes the links into the cluster that stores the requested block.

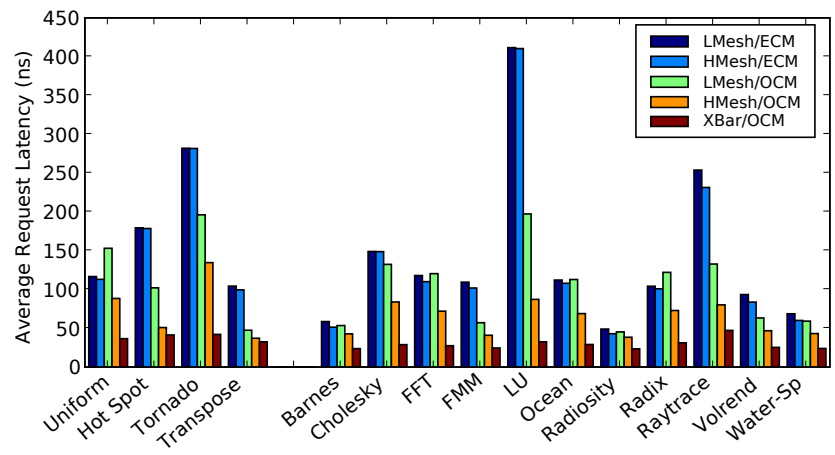

Figure 10: Average L2 Miss Latency

Figure 10 reports the average latency of an L2 cache miss to main memory. An L2 miss may be delayed in waiting for 
crossbar arbitration (the token) and by flow-control (destination cluster buffers may be full) before an interconnect message is generated. Our latency statistics measure both queue waiting times and interconnect transit times. LU and Raytrace see considerable average latency in ECM systems; it is improved dramatically by OCM and improved further by the optical crossbar. Note that the average latency can be high even when overall bandwidth is low when traffic is bursty.

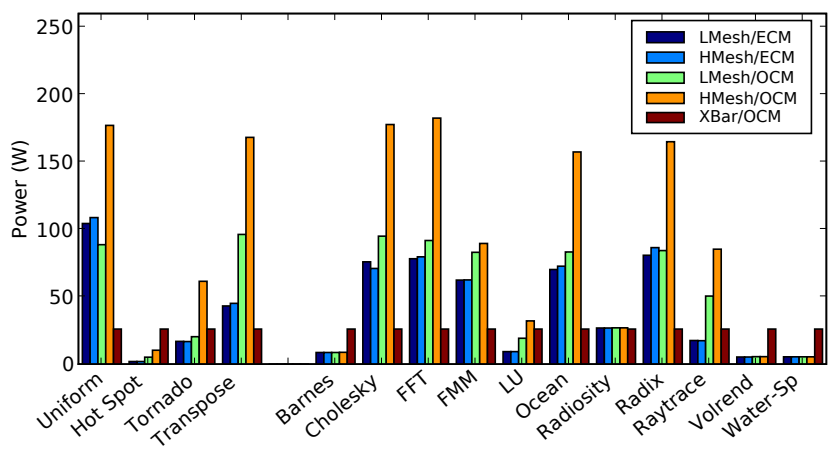

Figure 11: On-chip Network Power

Figure 11 shows the on-chip network dynamic power. For applications that fit in the L2 cache, the photonic crossbar can dissipate more power than for the electronic meshes (albeit ignoring mesh leakage power). However, for applications with significant memory demands, the power of the electronic meshes can fast become prohibitive with power of $100 \mathrm{~W}$ or more, even while providing lower performance.

\section{Related Work}

Recent proposals for optical networks in chip multiprocessors include a hierarchical multi-bus with optical global layer [15] and an optical, circuit-switched, mesh-based network managed by electrical control packets [28]. In contrast, our crossbar interconnect uses optical arbitration and control.

Optical crossbars have been proposed for Asynchronous Transfer Mode (ATM) switches [32] and for communication within processor clusters in large distributed shared memory systems [33]. This work relies on expensive VCSELs and free-space optical gratings to demultiplex the crossbar's wavelengths, unlike our solution which can be integrated into a modern 3D chip stack.

Most recent optical CMP interconnect proposals rely on electrical arbitration techniques $[6,15,28]$. Optical arbitration techniques have been investigated in SMP and ATM designs $[8,21]$. While these techniques employ token rings, their tokens circulate more slowly, as they are designed to stop at every node in the ring, whether or not the node is participating in the arbitration.
Chang et al. [5] overlay a 2D mesh CMP interconnect with a radio frequency interconnect to provide low latency shortcuts. They suggest frequency division multiple access similar to our DWDM to provide multiple channels per waveguide. Capacitive [10] and inductive [23] coupling technologies can provide wireless chip-to-chip communication which can be used within a package.

The 8- and 16-core Sun Niagara [17] and Niagara2 [24] chips use electrical crossbars. The 80-core Intel Polaris chip [31] and the 64-core MIT Raw processor [29] connect their cores with 2D mesh networks. A 2D mesh is easily laid out, regardless of its size. Latency for nonlocal traffic is high because multiple hops are required to communicate between cores unless they are physically adjacent. Random traffic is choked by the limited bisection bandwidth of a mesh $\left(O(n)\right.$ in an $n^{2}$-node mesh). Express Virtual Channels (EVCs) [18] alleviate the per-hop latency of packet based mesh and torus networks, but the paths cannot be arbitrarily shaped.

\section{Conclusions}

Over the coming decade, memory and inter-core bandwidths must scale by orders of magnitude to support the expected growth in per-socket core performance resulting from increased transistor counts and device performance. We believe recent developments in nanophotonics can be crucial in providing required bandwidths at acceptable power levels.

To investigate the potential benefits of nanophotonics on computer systems we have developed an architectural design called Corona. Corona uses optically connected memories (OCMs) that have been architected for low power and high bandwidth. A set of 64 OCMs can provide $10 \mathrm{~TB} / \mathrm{s}$ of memory bandwidth through 128 fibers using dense wavelength division multiplexing. Once this memory bandwidth comes on chip, the next challenge is getting each byte to the right core out of the hundreds on chip. Corona uses a photonic crossbar with optical arbitration to fully interconnect its cores, providing near uniform latency and $20 \mathrm{~TB} / \mathrm{s}$ of on-stack bandwidth.

We simulated a 1024 thread Corona system running synthetic benchmarks and scaled versions of the SPLASH2 benchmark suite. We found systems using opticallyconnected memories and an optical crossbar between cores could perform 2 to 6 times better on memory-intensive workloads than systems using only electrical interconnects, while dissipating much less interconnect power. Thus we believe nanophotonics can be a compelling solution to both the memory and network-on-chip bandwidth walls, while simultaneously ameliorating the power wall. 


\section{Acknowledgments}

We thank Ayose Falcon, Paolo Faraboschi, and Daniel Ortega for help with our COTSon simulations. We also thank Mikko Lipasti and Gabriel Black for their invaluable support and feedback. Dana Vantrease was supported in part by a National Science Foundation Graduate Research Fellowship.

\section{References}

[1] Proceedings of the ISSCC Workshop F2: Design of 3DChipstacks. IEEE, Feb 2007. Organizers: W. Weber and W. Bowhill.

[2] ANSI/IEEE. Local Area Networks: Token Ring Access Method and Physical Layer Specifications, Std 802.5. Technical report, 1989.

[3] K. Asanovic, et al. The Landscape of Parallel Computing Research: A View from Berkeley. Technical Report UCB/EECS-2006-183, EECS Department, University of California, Berkeley, Dec 2006.

[4] N. L. Binkert, R. G. Dreslinski, L. R. Hsu, K. T. Lim, A. G. Saidi, and S. K. Reinhardt. The M5 Simulator: Modeling Networked Systems. IEEE Micro, 26(4), Jul/Aug 2006.

[5] M.-C. F. Chang, J. Cong, A. Kaplan, M. Naik, G. Reinman, E. Socher, and S.-W. Tam. CMP Network-on-Chip Overlaid With Multi-Band RF-Interconnect. In HPCA, Feb 2008.

[6] H. J. Chao, K.-L. Deng, and Z. Jing. A Petabit Photonic Packet Switch (P3S). In INFOCOM, 2003.

[7] S. Chaudhry, P. Caprioli, S. Yip, and M. Tremblay. HighPerformance Throughput Computing. IEEE Micro, 25(3), May/Jun 2005.

[8] J. Choi, H. Lee, H. Hong, H. Kim, K. Kim, and H. Kim. Distributed optical contention resolution using an optical tokenring. Photonics Technology Letters, IEEE, 10(10), Oct 1998.

[9] W. Dally and C. Seitz. Deadlock-Free Message Routing in Multiprocessor Interconnection Networks. IEEE Transactions on Computers, C-36(5), May 1987.

[10] R. Drost, R. Hopkins, R. Ho, and I. Sutherland. Proximity Communication. JSSC, 39(9), Sep 2004.

[11] A. Falcon, P. Faraboschi, and D. Ortega. Combining Simulation and Virtualization through Dynamic Sampling. In ISPASS, Apr 2007.

[12] Intel. Intel Atom Processor. http://www.intel.com/technology/atom.

[13] - Introducing the $45 \mathrm{~nm}$ Next Generation Intel Core Microarchitecture. http://www.intel.com/technology/magazine/ 45nm/coremicroarchitecture-0507.htm.

[14] N. E. Jerger, L.-S. Peh, and M. H. Lipasti. Virtual Circuit Tree Multicasting: A Case for On-Chip Hardware Multicast Support. ISCA, Jun 2008.

[15] N. Kirman, M. Kirman, R. K. Dokania, J. F. Martinez, A. B. Apsel, M. A. Watkins, and D. H. Albonesi. Leveraging Optical Technology in Future Bus-based Chip Multiprocessors. In MICRO, 2006.
[16] B. R. Koch, A. W. Fang, O. Cohen, and J. E. Bowers. Modelocked silicon evanescent lasers. Optics Express, 15(18), Sep 2007.

[17] P. Kongetira, K. Aingaran, and K. Olukotun. Niagara: A 32-Way Multithreaded Sparc Processor. IEEE Micro, 25(2), 2005.

[18] A. Kumar, L.-S. Peh, P. Kundu, and N. K. Jha. Express Virtual Channels: Towards the Ideal Interconnection Fabric. In ISCA, Jun 2007.

[19] R. Kumar, V. Zyuban, and D. M. Tullsen. Interconnections in Multi-Core Architectures: Understanding Mechanisms, Overheads and Scaling. In ISCA, Jun 2005.

[20] M. Lipson. Guiding, Modulating, and Emitting Light on Silicon-Challenges and Opportunities. Journal of Lightwave Technology, 23(12), Dec 2005.

[21] A. Louri and A. K. Kodi. SYMNET: An Optical Interconnection Network for Scalable High-Performance Symmetric Multiprocessors. Applied Optics, 42(17), Jun 2003.

[22] M. M. K. Martin, D. J. Sorin, M. D. Hill, and D. A. Wood. Bandwidth Adaptive Snooping. In HPCA, Feb 2002.

[23] N. Miura, D. Mizoguchi, T. Sakurai, and T. Kuroda. Analysis and Design of Inductive Coupling and Transceiver Circuit for Inductive Inter-Chip Wireless Superconnect. JSSC, 40(4), Apr 2005.

[24] U. Nawathe, M. Hassan, L. Warriner, K. Yen, B. Upputuri, D. Greenhill, A. Kumar, and H. Park. An 8-Core 64-Thread 64b Power-Efficient SPARC SoC. In ISSCC, Feb 2007.

[25] R. Palmer, J. Poulton, W. J. Dally, J. Eyles, A. M. Fuller, T. Greer, M. Horowitz, M. Kellam, F. Quan, and F. Zarkeshvarl. A $14 \mathrm{~mW} 6.25 \mathrm{~Gb} / \mathrm{s}$ Transceiver in $90 \mathrm{~nm}$ CMOS for Serial Chip-to-Chip Communications. In ISSCC, Feb 2007.

[26] L. Schares, et al. Terabus: Terabit/Second-Class Card-Level Optical Interconnect Technologies. IEEE Journal of Selected Topics in Quantum Electronics, 12(5), Sep/Oct 2006.

[27] Semiconductor Industries Association. International Technology Roadmap for Semiconductors. http://www.itrs.net/, 2006 Update.

[28] A. Shacham, B. G. Lee, A. Biberman, K. Bergman, and L. P. Carloni. Photonic NoC for DMA Communications in Chip Multiprocessors. In IEEE Hot Interconnects, Aug 2007.

[29] M. B. Taylor, et al. Evaluation of the Raw Microprocessor: An Exposed-Wire-Delay Architecture for ILP and Streams. In ISCA, Jun 2004.

[30] S. Thoziyoor, N. Muralimanohar, J. Ahn, and N. P. Jouppi. CACTI 5.1. Technical Report HPL-2008-20, HP Labs.

[31] S. Vangal, et al. An 80-Tile 1.28TFLOPS Network-on-Chip in 65nm CMOS. In ISSCC, Feb 2007.

[32] B. Webb and A. Louri. All-Optical Crossbar Switch Using Wavelength Division Multiplexing and Vertical-Cavity Surface-Emitting Lasers . Applied Optics, 38(29), Oct 1999.

[33] —. A Class of Highly Scalable Optical CrossbarConnected Interconnection Networks (SOCNs) for Parallel Computing Systems. TPDS, 11(5), 2000.

[34] S. C. Woo, M. Ohara, E. Torrie, J. P. Singh, and A. Gupta. The SPLASH-2 Programs: Characterization and Methodological Considerations. In ISCA, Jun 1995.

[35] Q. Xu, B. Schmidt, S. Pradhan, and M. Lipson. Micrometrescale silicon electro-optic modulator. Nature, 435, May 2005. 